\title{
Advanced gastric cancer with a duplicated hepatic artery: preoperative diagnostic value of multidetector-row computed tomography for surgical resection
}

\author{
Masanori Tokunaga, Shigekazu Ohyama, Souya Nunobe, Naoki Hiki, Tetsu Fukunaga, Yasuyuki Seto, \\ and Toshiharu Yamaguchi
}

Department of Gastroenterological Surgery, Cancer Institute Hospital, 3-10-6 Ariake, Koto-ku, Tokyo 135-8550, Japan

\begin{abstract}
A 63-year-old woman with appetite loss and general fatigue underwent gastrointestinal fiberscopy, which revealed type 2 advanced gastric cancer. Multidetector-row computed tomography revealed a massive gastric cancer invading the left hepatic lobe, pancreatic head, and common hepatic artery, as well as revealing a duplicated hepatic artery in which the right hepatic artery branched directly from the celiac axis, and ran behind the splenic vein. On the other hand, the common hepatic artery ran anterior to the splenic vein. We were able to perform pancreaticoduodenectomy with common hepatic artery resection and left lobectomy as curative surgery because her duplicated hepatic artery enabled us to ligate the common hepatic artery. Her postoperative clinical course was uneventful, and she is in good health 3 years after the surgery, without recurrence. We consider that multidetector-row computed tomography is very useful for the diagnosis of vascular anomaly, preoperative staging and decision making on the appropriate surgical strategy.
\end{abstract}

Key words Duplicated hepatic artery - MDCT - Gastric cancer $\cdot$ Pancreaticoduodenectomy

\section{Introduction}

Surgical resection with curative intent is mandatory for the long-term survival of patients with advanced gastric cancer. Recently, many chemotherapy regimens have been proven to be effective for treating advanced gastric cancer; however, the complete response rate is very low even with aggressive therapies, and long-term survival is anecdotal [1,2]. Consequently, curative resection remains the principal treatment for advanced gastric cancer.

Offprint requests to: $\mathrm{M}$. Tokunaga

Received: November 26, 2006 / Accepted: May 30, 2007
In patients with $\mathrm{T} 4$ gastric cancer, curative surgery can be achieved with concomitant resection of the invaded organs. However, curative resection is not necessarily possible in all cases. In patients with advanced gastric cancer with infiltration of the pancreatic head and common hepatic artery (CHA), curative surgery is usually impossible, because pancreaticoduodenectomy (PD) with $\mathrm{CHA}$ resection leads to an abrupt reduction in the arterial blood supply to the liver and results in severe liver damage. We experienced a case of advanced gastric cancer that infiltrated these organs, i.e., the pancreatic head and CHA. We were able to perform curative surgery in this patient because preoperative multidetector-row computed tomography (MDCT) revealed the presence of a duplicated hepatic artery. Here, we present a very rare case of advanced gastric cancer in a patient with a duplicated hepatic artery, and we note the value of MDCT for preoperative diagnosis.

\section{Case report}

A 63-year-old Japanese woman presented to her family physician with appetite loss and general fatigue in June 2003. Gastrointestinal fiberscopy and upper gastrointestinal (GI) series revealed type 2 advanced gastric cancer in the antrum, with pyloric stenosis and duodenal invasion (Fig. 1). The patient was referred to the Cancer Institute Hospital for further examination. Laboratory examination revealed severe anemia (hemoglobin, $8.0 \mathrm{~g} /$ dl) and poor nutritional condition (albumin, $3.3 \mathrm{~g} / \mathrm{dl}$ ). Abdominal MDCT revealed massive gastric cancer directly invading the left hepatic lobe and pancreatic head as well as vascular anomaly. Metastasized lymph nodes also invaded the CHA directly (Fig. 2). Although paraaortic lymph nodes were identified, CT did not reveal any distant metastasis. In general, PD with $\mathrm{CHA}$ resection is considered to be impossible, because it 


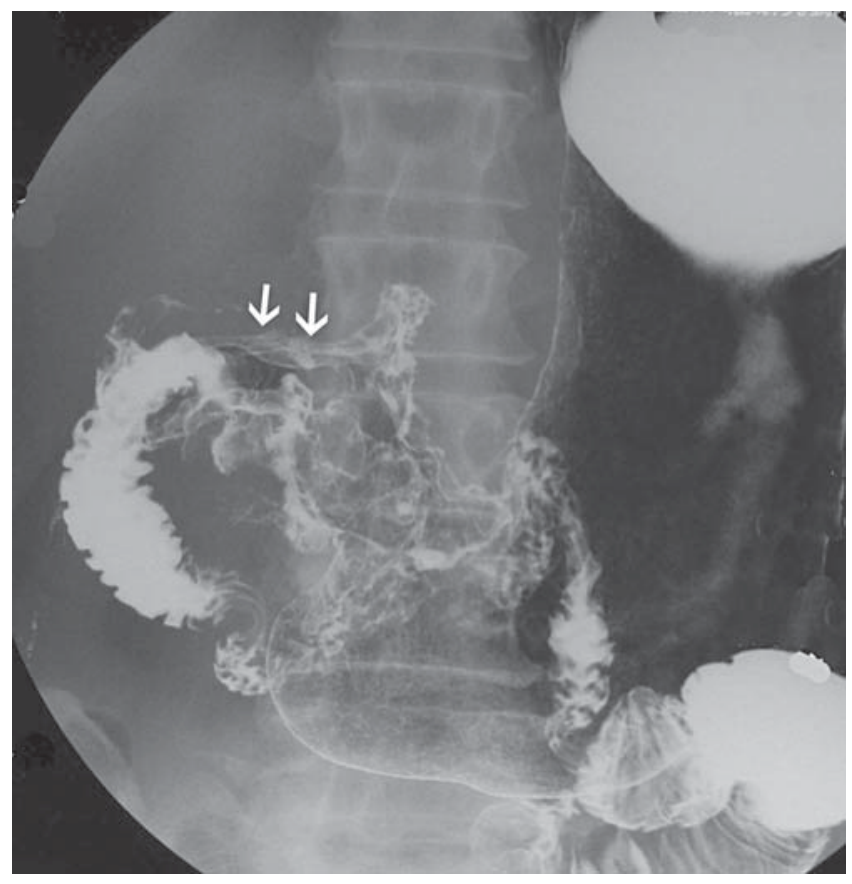

Fig. 1. Upper gastrointestinal (GI) series revealed type 2 advanced gastric cancer invading the duodenum (arrows)

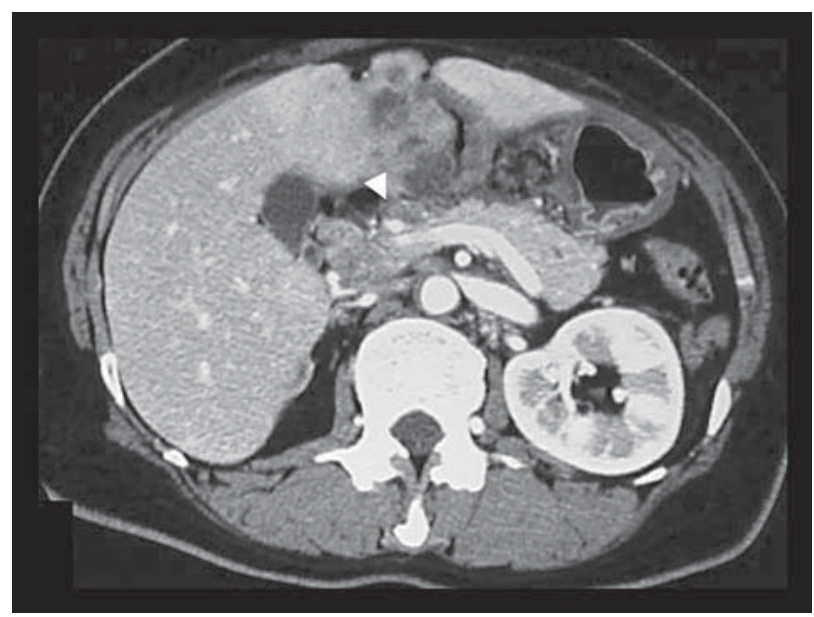

Fig. 2. The left lobe was directly invaded by a huge gastric tumor. Metastasized lymph nodes also invaded the common hepatic artery directly (white arrowhead)

results in an abrupt reduction in the arterial blood supply to the liver, thereby leading to severe liver damage. In the present patient, the MDCT had also revealed a vascular anomaly. The right hepatic artery branched directly from the celiac axis and ran behind the splenic vein. On the other hand, the CHA ran in its usual position, running into the gastroduodenal artery, left hepatic artery, and right gastric artery (Fig. 3A-D). Although the CHA was invaded by the metastasized perigastric lymph nodes, the right hepatic artery was intact. In this patient, we considered that the arterial blood supply to the right hepatic lobe would be preserved after PD with CHA resection. Therefore, we assumed that surgical resection could be performed with PD that included CHA resection and left lobectomy. The surgery was performed on August 18, 2003, after obtaining written informed consent from the patient.

During laparotomy, the massive tumor that occupied the upper abdomen was easily detected and was found to directly invade the left hepatic lobe. The metastasized perigastric lymph nodes involved the pancreatic head and CHA. The duodenum was also affected. The patient did not have peritoneal dissemination, and the result of peritoneal lavage fluid cytology was negative for cancer. Macroscopically, the right hepatic artery was not invaded by gastric cancer. We considered that curative surgery could be performed while preserving the arterial blood supply to the right hepatic lobe. Subsequently, PD with CHA resection and extended lymph node dissection was performed (Fig. 4). The left hepatic lobe was also resected due to direct invasion and loss of arterial blood supply. The operation duration was $445 \mathrm{~min}$, and the estimated blood loss was $1450 \mathrm{ml}$. The patient received a transfusion due to bleeding during surgery and preoperative anemia. Her postoperative course was uneventful, except for minor pancreatic leakage that was controlled by drainage under local anesthesia and the continuing administration of antibiotics. The patient was discharged on postoperative day 23.

The resected specimen showed type 2 advanced gastric cancer that directly invaded the left hepatic lobe (Fig. 5). The metastasized regional lymph nodes also invaded the pancreatic head and CHA. Of the 48 examined lymph nodes, 17 were cancer-positive, and some of these were paraaortic lymph nodes. The final diagnosis was stage IV gastric cancer, designated T4N3M0 according to the Japanese classification of gastric carcinoma [3].

The patient underwent adjuvant chemotherapy with S-1 for 1 year, and is in good health 3 years after the surgery, without recurrence.

\section{Discussion}

Recent advances in imaging systems have resulted in a major change in the preoperative evaluation of patients undergoing gastric cancer surgery.

First, the development of MDCT and other imaging modalities such as endoscopic ultrasound examination has facilitated the correct diagnosis of gastric cancer prior to surgery. Many authors have reported the usefulness of these imaging modalities for preoperative 

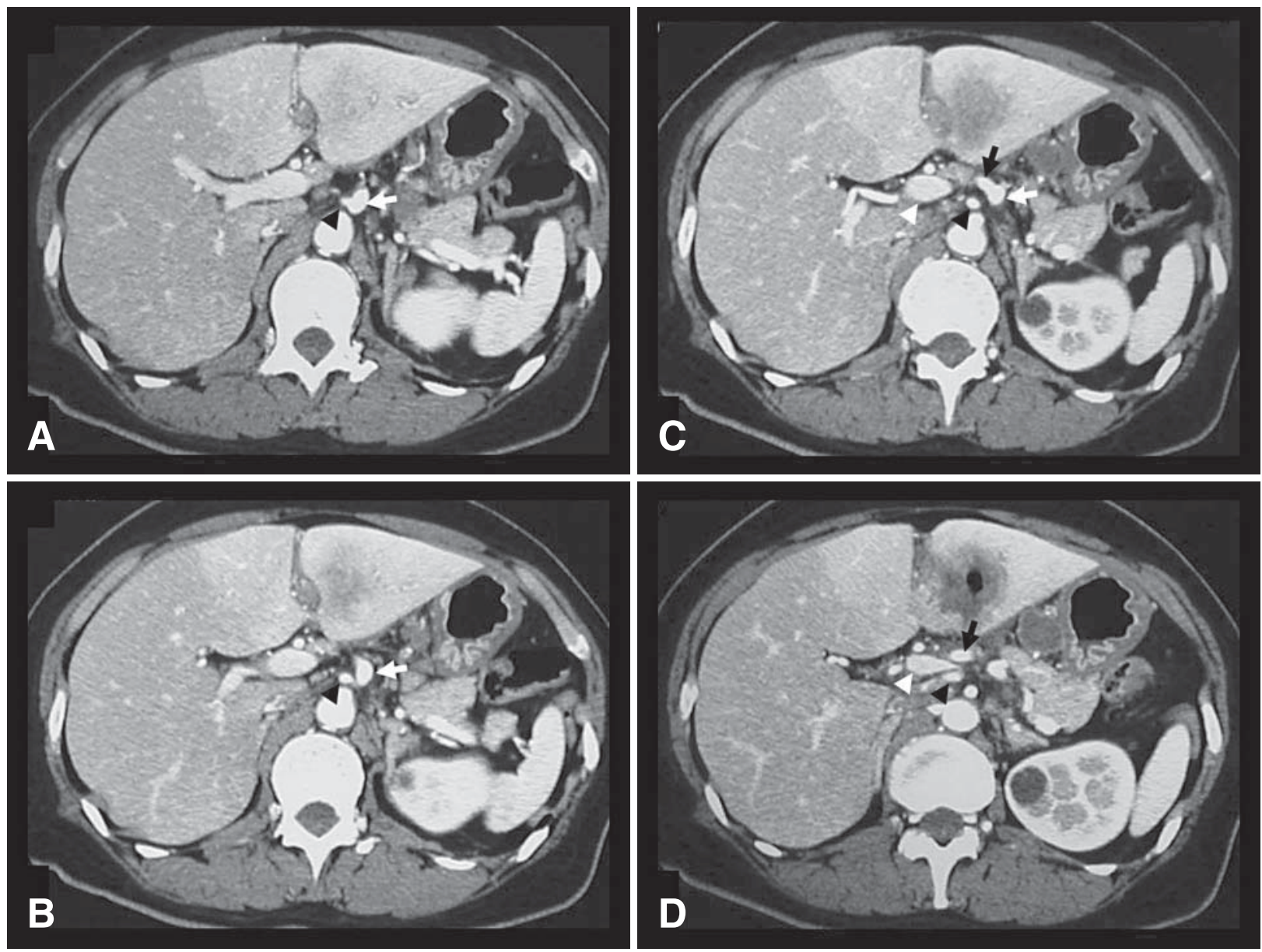

Fig. 3A-D. Multidetector-row computed tomography revealed a duplicated hepatic artery. The right hepatic artery (black arrowheads; $\mathbf{A}, \mathbf{B}, \mathbf{C}, \mathbf{D}$ ) branched from the celiac axis (white arrows; $\mathbf{A}, \mathbf{B}, \mathbf{C}$ ) directly and ran behind the splenic vein (white arrowheads; $\mathbf{C}, \mathbf{D})$. The common hepatic artery (black arrows; $\mathbf{C}, \mathbf{D}$ ) ran in its usual position, i.e., anterior to the splenic vein

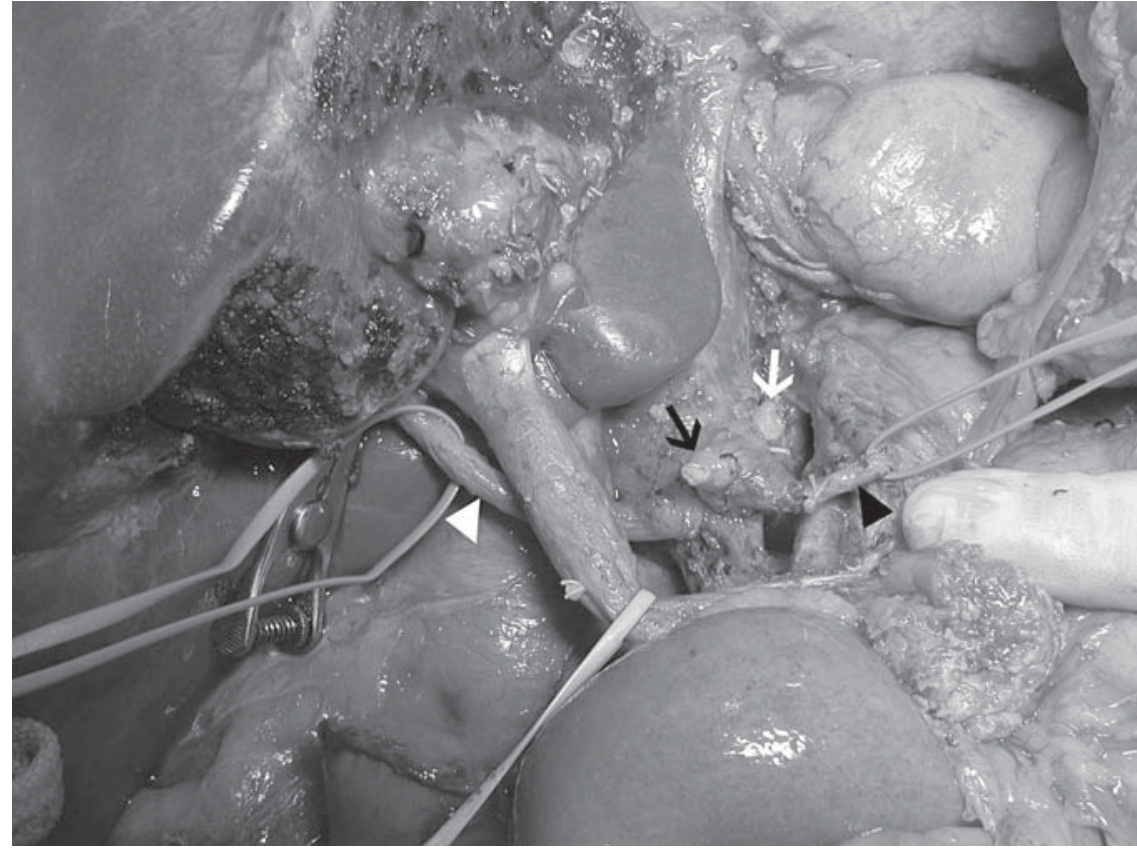

Fig. 4. The left gastric artery (white arrow) and common hepatic artery (black arrow) were ligated at their origin. The right hepatic artery (white arrowhead) and splenic artery (black arrowhead) were preserved. The right hepatic artery ran behind the splenic vein 


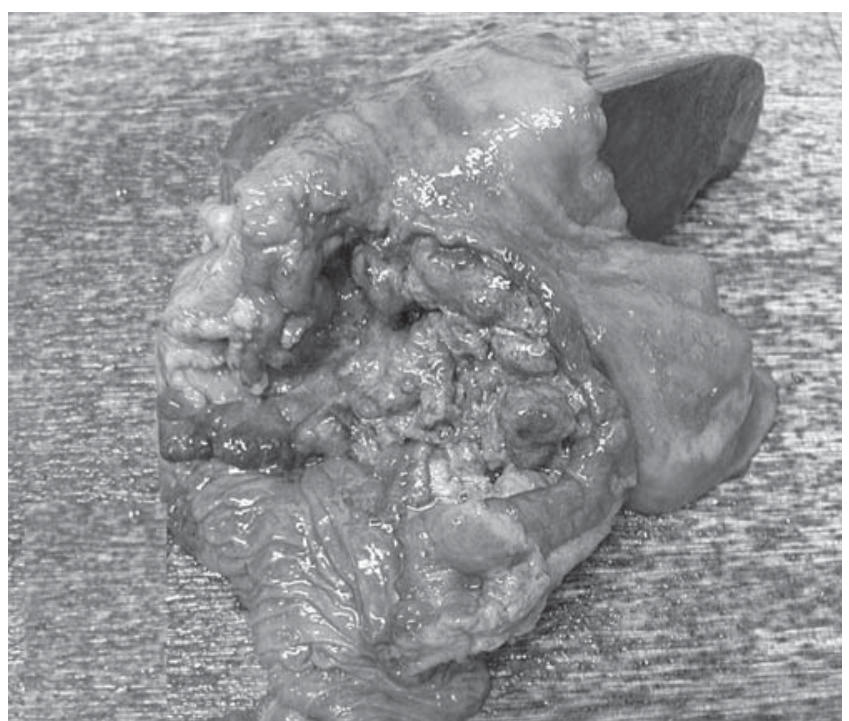

Fig. 5. The resected specimen showed a massive type 2 gastric cancer invading the left hepatic lobe

staging, and accurate staging by MDCT can aid in preventing unnecessary surgeries and in safely performing essential surgeries [4-7].

Second, MDCT is useful for evaluating the vascular anatomy. Prior to MDCT, angiography was the only modality available for this; however, it could not be easily performed because of its invasiveness. Currently, MDCT is preferred over angiography for the evaluation of the vascular anatomy because it is less invasive. We previously reported the usefulness of preoperative MDCT for the diagnosis of Adachi type VI vascular anomaly [8]. In this anomaly, the CHA branches from the superior mesenteric artery (SMA) and runs posterior to the splenic vein, thereby making it difficult to trace the CHA at the superior border of the pancreas [8]. It is sometimes difficult to dissect the no. 8a lymph nodes in patients with this anomaly. The usefulness of determining the origin of the posterior gastric artery and the gastrocolic trunk has been reported, and these determinations may aid in the dissection of the no. 6 and no. 11p lymph nodes, respectively [9-11]. In the present patient, a vascular anomaly was diagnosed, in which the right hepatic artery branched directly from the celiac axis and ran posterior to the splenic vein; this vascular branching pattern made surgical resection possible. This anomaly is known as a duplicated hepatic artery, and its frequency has been reported to be $1 \%-$ $5 \%[12]$.

In the present patient, 4-slice MDCT was performed; however, a three-dimensional (3D) angiographic image was not obtained because 3D angiography was not routinely performed at that time. The quality of 3D angiographic images obtained using 4-slice MDCT was not as good as that obtained using 16-slice or 64-slice MDCTs. This was the reason for the previously limited use of $3 \mathrm{D}$ angiography. Currently, 16-slice MDCT is generally used in preoperative examinations prior to gastric cancer surgery, and a superior 3D angiographic image is obtained easily in our institute. The present case demonstrated that 4-slice MDCT was sufficiently effective in evaluating the vascular pattern and related anomalies; however, it is believed that the more advanced MDCT techniques such as 16-slice or 64-slice are superior to the 4-slice MDCT technique in the evaluation of vascular anomalies and their preoperative diagnosis.

Despite remarkable recent advances in surgical techniques and chemotherapies, the prognosis of advanced gastric cancer remains poor. In patients with T4 gastric cancer, the 5-year survival rate after curative surgery is only $16.8 \%-32.2 \%$ [13-17]. Despite the low survival rates, many authors recommend surgical resection, which is the only option for the long-term survival of these patients. However, the morbidity and mortality rates of surgery are higher for advanced gastric cancer than for early gastric cancer. In addition, curative surgery for T4 gastric cancer is not necessarily possible in all cases.

In our patient, the presence of a duplicated hepatic artery enabled us to perform an extended surgery including PD with left lobectomy, CHA resection, and extended lymph node dissection. The efficacy of PD in treating advanced gastric cancer has not been proved. Some authors have reported a 5-year survival rate in patients with advanced gastric cancer after PD; however, the morbidity and mortality rates of PD were higher than those of conventional gastrectomy [18-21]. Saka et al. [18] reported 23 patients with advanced gastric cancer who underwent PD at the same institute. The 5 -year survival rate after PD was $47 \%$ in patients without incurable factors. Hirose et al. [19] reported on 10 patients with gastric cancer who underwent $\mathrm{PD}$, and they concluded that PD might improve the prognosis of gastric cancer that infiltrated the pancreatic head. Although the morbidity rate was reported to be high in both these studies $[18,19]$, the mortality rate was zero.

The efficacy of paraaortic lymph node dissection is debatable [22-24]. Since Japanese surgeons have believed that complete resection is essential for curing advanced gastric cancer, extended lymph node dissection (as in the present patient), is preferred and widely performed. Kunisaki et al. [24] have reported that paraaortic lymph node dissection may be beneficial for patients with advanced $\mathrm{pN} 2$ gastric cancer with tumors measuring from 50 to $100 \mathrm{~mm}$. However, a large randomized controlled trial performed in Japan (JCOG 9501) to compare D2 and D3 dissection in patients with $\mathrm{T} 2$ or T3 advanced gastric cancer revealed that prophylactic paraaortic lymph node dissection was not effec- 
tive and did not result in long-term survival. Therefore, the therapeutic application of paraaortic lymph node dissection for treating advanced gastric cancer is rapidly decreasing. In our patient, surgical resection was the only option for cure and for ensuring long-term survival; hence, PD with left lobectomy and CHA resection was performed. Paraaortic lymph node dissection was also performed, because the result of JCOG 9501 had not been clarified.

Currently, extended surgery for treating extremely advanced cases of gastric cancer is controversial. However, in particular patients, curative surgery can be attempted because this extended surgery may lead to long-term survival. Additionally, MDCT should be performed prior to surgery to evaluate the vascular anatomy; this may aid in selecting the appropriate treatment method and in safely performing the surgery.

Acknowledgment We are deeply indebted to Noriko Okita for checking the English in this article.

\section{References}

1. Ajani JA. Evolving chemotherapy for advanced gastric cancer. Oncologist 2005;10(Suppl 3):49-58.

2. Sastre J, Garcia-Saenz JA, Diaz-Rubio E. Chemotherapy for gastric cancer. World J Gastroenterol 2006;12:204-13.

3. Japanese Gastric Cancer Association. Japanese classification of gastric carcinoma, 2nd English edition. Gastric Cancer 1998;1: $10-24$.

4. Blackshaw GR, Stephens MR, Lewis WG, Boyce J, Barry JD, Edwards P, et al. Progressive CT system technology and experience improve the perceived preoperative stage of gastric cancer. Gastric Cancer 2005;8:29-34.

5. Shinohara T, Ohyama S, Yamaguchi T, Muto T, Kohno A, Kato $\mathrm{Y}$, et al. Clinical value of multidetector row computed tomography in detecting lymph node metastasis of early gastric cancer. Eur J Surg Oncol 2005;31:743-8.

6. Kim AY, Kim HJ, Ha HK. Gastric cancer by multidetector row CT: preoperative staging. Abdom Imaging 2005;30:465-72.

7. Habermann CR, Weiss F, Riecken R, Honarpisheh H, Bohnacker S, Staedtler C, et al. Preoperative staging of gastric adenocarcinoma: comparison of helical CT and endoscopic US. Radiology 2004;230:465-71.

8. Tokunaga M, Ohyama S, Hiki N, Fukunaga T, Seto Y, Yamaguchi T. Five cases of gastric cancer with Adachi type VI vascular anomaly in which preoperative diagnosis was made by MDCT and 3D-angiography (in Japanese). J Jpn Surg Assoc 2006;67: 2604-8.

9. Matsuki M, Tanikake M, Kani H, Tatsugami F, Kanazawa S, Kanamoto T, et al. Dual-phase 3D CT angiography during a single breath-hold using 16-MDCT: assessment of vascular anatomy before laparoscopic gastrectomy. AJR Am J Roentgenol 2006;186:1079-85.

10. Okabayashi T, Kobayashi M, Morishita S, Sugimoto T, Akimori $\mathrm{T}$, Namikawa $\mathrm{T}$, et al. Confirmation of the posterior gastric artery using multi-detector row computed tomography. Gastric Cancer 2005;8:209-13.

11. Matsuki M, Kani H, Tatsugami F, Yoshikawa S, Narabayashi I, Lee SW, et al. Preoperative assessment of vascular anatomy around the stomach by 3D imaging using MDCT before laparoscopy-assisted gastrectomy. AJR Am J Roentgenol 2004; 183:145-51.

12. Fasel JH, Muster M, Gailloud P, Mentha G, Terrier F. Duplicated hepatic artery: radiologic and surgical implications. Acta Anat (Basel) 1996;157:164-8.

13. Carboni F, Lepiane P, Santoro R, Lorusso R, Mancini P, Sperduti I, et al. Extended multiorgan resection for T4 gastric carcinoma: 25-year experience. J Surg Oncol 2005;90:95-100.

14. Kunisaki C, Akiyama H, Nomura M, Matsuda G, Otsuka Y, Ono $\mathrm{HA}$, et al. Surgical outcomes in patients with T4 gastric carcinoma. J Am Coll Surg 2006;202:223-30.

15. Saito H, Tsujitani S, Maeda Y, Fukuda K, Yamaguchi K, Ikeguchi $\mathrm{M}$, et al. Combined resection of invaded organs in patients with T4 gastric carcinoma. Gastric Cancer 2001;4:206-11.

16. Dhar DK, Kubota H, Tachibana M, Kinugasa S, Masunaga R, Shibakita M, et al. Prognosis of T4 gastric carcinoma patients: an appraisal of aggressive surgical treatment. J Surg Oncol 2001;76: 278-82.

17. Kobayashi A, Nakagohri T, Konishi M, Inoue K, Takahashi S, Itou M, et al. Aggressive surgical treatment for T4 gastric cancer. J Gastrointest Surg 2004;8:464-70.

18. Saka M, Mudan SS, Katai H, Sano T, Sasako M, Maruyama K. Pancreaticoduodenectomy for advanced gastric cancer. Gastric Cancer 2005;8:1-5.

19. Hirose K, Onchi H, Iida A, Katayama K, Yamaguchi A, Nakagawara G. Surgical results of pancreaticoduodenectomy for carcinoma of the distal third of the stomach. Int Surg 1999;84:18-24.

20. Ajisaka H, Fujita H, Kaji M, Maeda K, Yabushita K, Konishi K, et al. Treatment of patients with gastric cancer and duodenal invasion. Int Surg 2001;86:9-13.

21. Menjo M, Nimura Y, Hayakawa N, Kamiya J, Kondo S, Nagino $\mathrm{M}$, et al. Ten-year survival after pancreatoduodenectomy for advanced gastric cancer - report of two cases. Hepatogastroenterology 1999;46:1253-6.

22. Nakane Y, Okamura S, Masuya Y, Okumura S, Akehira K, Hioki $\mathrm{K}$. Incidence and prognosis of para-aortic lymph node metastasis in gastric cancer. Hepatogastroenterology 1998;45:1901-6.

23. Maeta M, Yamashiro H, Saito H, Katano K, Kondo A, Tsujitani S, et al. A prospective pilot study of extended (D3) and superextended para-aortic lymphadenectomy (D4) in patients with T3 or T4 gastric cancer managed by total gastrectomy. Surgery 1999;125:325-31.

24. Kunisaki C, Akiyama H, Nomura M, Matsuda G, Otsuka Y, Ono $\mathrm{H}$, et al. Comparison of surgical results of D2 versus D3 gastrectomy (para-aortic lymph node dissection) for advanced gastric carcinoma: a multi-institutional study. Ann Surg Oncol 2006;13: 659-67. 